\title{
9
}

Josep-Antoni Ybarra*

\section{ARGELIA DISEÑANDO SU DESARROLLO}

Desde el inicio del siglo XXI, Argelia ha puesto un especial énfasis en llevar a cabo un proceso de transformación económica que le permita mejorar el nivel de vida de su población, así como el diversificar su economía excesivamente centrada hasta ahora en la explotación de sus recursos energéticos. Para ello ha propuesto una serie de planes de desarrollo que manifiestan claramente dos intenciones: en primer lugar se pretende llevar a cabo un cambio y una notable mejora en las infraestructuras sociales (viviendas, transporte, enseñanza, sanidad, etcétera); y en segundo lugar, el propósito es el de modificar su excesiva especialización productiva poniendo en marcha nuevas oportunidades para sectores como la agricultura, las energías alternativas y el turismo. Se finaliza el escrito apuntando algunos de los problemas que pueden limitar estas intenciones.

Palabras clave: Argelia, planes de desarrollo, transformación económica.

Clasificación JEL: O10, O20, O55.

\section{Una ojeada en general}

Con una población de 40 millones de habitantes ${ }^{1}$, algo inferior a la de España, Argelia es el país más extenso de África; con una extensión semejante a casi cinco veces la de España. Dos aspectos son los que fundamentalmente condicionan su devenir económico: en primer lugar la posición geográfica que ocupa en el norte de África, y en segundo lugar, la abundancia de recursos naturales estratégicos que posee en su territorio (Aissaoui, 2001); ello determina en gran parte su comportamiento económico. Sus reservas de gas se estiman en 159,1 Tcf (trillion cubic feet) (décimo país del mundo en reservas de gas) lo que significa que con el actual grado de extracción, Argelia tardaría 60 años en agotar sus reservas. En cuanto a petróleo, su posición mundial es un tanto más discreta ya que ocupa

* Universidad de Alicante.

Versión de febrero de 2016.

1 Los datos que aparecen a lo largo del texto, y salvo que se indique expresamente lo contrario, tienen como fuente las del Banco Mundial y las del Fondo Monetario Internacional, publicaciones que se citan en la bibliografía al final del texto. la posición decimocuarta en el rango mundial, con un volumen estimado de 11.800 millones de barriles, que a un ritmo de extracción semejante al actual, se agotaría en unos 18-20 años vista. Situado en el norte de África, en el Magreb mediterráneo, puede representar una puerta de entrada hacia Oriente Medio y África, de la misma manera que ocupa una posición geoestratégica de primer orden en cuanto a sus relaciones con Europa.

Para entender su comportamiento económico, a esta posición de privilegio en el Mediterráneo y a sus recursos naturales, hay que agregarle un hecho que le viene determinado por los condicionamientos históricos que tuvo que atravesar, los cuales han dejado una forma peculiar de entender cómo comportarse y funcionar en el ámbito económico. Así ocurre con el hecho de haber sido colonia francesa durante 130 años (hasta 1962) y posteriormente el haber tenido una aproximación política y económica a la antigua URSS. Derivado de ello, se instaura como norma el diseño de una forma de Gobierno próximo a lo que podría ser una economía administrada, $D$ 
tutelada por los dirigentes que pueda tener en cada momento.

Los dos aspectos que destacan de manera reiterada y que han venido caracterizando a la economía argelina son: la existencia de unos recursos que le permiten obtener, de una manera relativamente sencilla, rentas regulares y seguras en los mercados internacionales, y el modo de gobernar, muy próximo a lo que podría ser una economía controlada por los grupos que en cada momento estuviesen próximos al Gobierno. Estos dos aspectos influyen sobremanera en el comportamiento de la economía y en la actitud de la sociedad argelina ante la economía, de tal forma que llegan a determinar la manera de cómo los recursos internos (capitales, trabajo, suelo), la regulación de la economía y las instituciones generales (enseñanza, sanidad, justicia, relaciones socioeconómicas) llegan a desenvolverse.

\section{Entre las transformaciones pasadas y los desafíos futuros}

Al inicio de la década de 1990 hubo una declaración de guerra por parte del Frente Islámico de Salvación (FIS) contra el Gobierno existente en aquellos momentos. EI FIS, que nació de una revuelta estudiantil en Argel, se consolida a lo largo de los años 1990 haciendo apariciones armadas de forma intermitente a lo largo de los años siguientes hasta que se llega a firmar la paz en 1999; es lo que se le conoce como el décennie noire. Ello supuso que de forma más o menos encubierta existiera una situación de inestabilidad social derivada del conflicto civil bélico silenciado que llega prácticamente hasta entrado el siglo XXI. Por ello, la vida y el devenir económico para la última generación de argelinos ha estado salpicada por una cierta incertidumbre debido a razones bélicas. Esto ha hecho que las oportunidades económicas que se planteaban tuviesen dificultades para llevarse a la práctica. Desde los años noventa del pasado siglo la tasa de crecimiento de la economía argelina ha sido ligeramente positiva, si bien el auténtico boom se produce en los últimos años (el crecimiento del PIB en 2014 ha sido del 4 por 100). De esta forma, ocurre que los últimos 30 años han trascurrido para Argelia en el marco de un constante dilema, entre la modernidad y la tradición, entre la apertura y la cerrazón y, en este sentido, las posibilidades que brotaban de las rentas procedentes de los recursos energéticos, no podían garantizar que pudieran derivarse de ellas efectos positivos y que se esparcieran sobre una sociedad anclada en la tradición (United Nations Development, 2011). También es cierto que el intento de modernización fue un hecho, y que esta modernización se pretendió que llegara al conjunto de la sociedad (este era uno de los objetivos prioritarios que reiteradamente han manifestado cualquiera de sus gobernantes en los últimos tiempos) (Mouffok, 2001). Esto ha permitido que la esperanza de vida de la última generación se haya alargado en cinco años (pasando de 66 a 71 años de vida), la escolarización primaria sea hoy prácticamente obligatoria y general para toda la población, y que la enseñanza secundaria aumenta en un 10 por 100 el número de jóvenes que se benefician de ello (al haber pasado de cubrir al 58 por 100 de los jóvenes a un 69 por 100); lo mismo puede observarse en la práctica totalidad de indicadores de bienestar (escuelas, hospitales, salubridad, acceso a agua, urbanización, carreteras, etcétera) y que informan sobre esa intención de modernizar el país.

El segundo de los desafíos que se han propuesto es el de dar un salto en lo que respecta a su especialización productiva. Argelia es un país excesivamente centrado en los recursos energéticos; su estructura productiva poco ha variado en los últimos treinta años; la industria -relacionada principalmente con el sector energético- era y es la actividad principal, ya que absorbe prácticamente el 50 por 100 de toda la actividad que se realiza (en cuanto a dedicación de la mano de obra y en cuanto a la aportación al PIB). Esto, si bien no es un obstáculo para el desarrollo, puede llegar a serlo en tanto que la facilidad en la obtención de rentas en $\triangleright$ 
las mercados internacionales puede hacer que ello derive en una incapacidad para impulsar otro tipo de actividades. Llega a ocurrir que, como resultado de una excesiva riqueza y por el deslumbramiento de aquellos recursos naturales, esta prosperidad en lugar de facilitar el posible desarrollo de otras actividades, lo que hace es contagiar una inoperancia al conjunto de la economía y de la sociedad, en tanto que la simplicidad en la obtención de rentas hace que el resto de sectores no se modifiquen; de esta forma el atraso puede alargarse en el tiempo y en el espacio, hacerse crónico y generalizarse, además de introducir otros aspectos relacionados con la corrupción, el despilfarro, el consumo excesivo y el aumento de la deuda.

¿Es este el caso de Argelia? En parte puede decirse que el encontrarse en un lugar privilegiado en el marco del comercio internacional con una ventaja competitiva derivada de aquellos recursos naturales -gas y petróleo- hace que la diversificación productiva no tuviese una prioridad inmediata; esto, a su vez, ha comportado que un aumento de la actividad general no se produzca. De esta forma, aparece una tasa de desocupación del 10 por 100, desocupación que empieza a ser preocupante en la población joven (la que va de los 18 a los 30 años), ya que se está convirtiendo en una población nini, llegando a presentar una tasa de desocupación de los jóvenes del 30 por 100 (Bardak, 2015).

Por ello, la diversificación productiva por un lado y la modernización del país por otro son los grandes retos que se propusieron y se continúan exigiendo para que Argelia pueda dar un salto hacia un futuro con mayores oportunidades para el conjunto de su población.

\section{Trabajo, empleo y empresas: las nuevas relaciones}

En el espacio temporal de 50 años, la población argelina se ha multiplicado por tres. Este hecho ha derivado en que aparezcan nuevas y mayores necesidades y que a su vez se requieran realizar cambios en sus estructuras productivas. Se precisa una mayor oferta de bienes y servicios, pero también que sus relaciones productivas y comerciales (empresariales, institucionales, sociales) se modifiquen con objeto de poder hacer frente a las nuevas exigencias. Estos cambios han ido dándose tímida y paulatinamente; así tenemos cómo desde 1978 hasta la actualidad se han promovido programas y leyes que abarcan tanto lo que son las políticas activas de fomento de empleo como las que van dirigidas a la creación y dinamización de las pequeñas empresas (gestión de microcréditos, servicios de apoyo a la creación de empresas, etcétera) (Bardak, 2013). Como resultado de todo ello está emergiendo una nueva estructura productiva que a su vez arrastra a una estructura ocupacional que puede resumirse en tres aspectos.

En primer lugar con una nueva oferta relacionada con los servicios públicos y las demandas sociales, como son la salud, la educación, la organización y la gestión de la Administración central y local. En estas actividades la mejora de la ocupación se ha podido constatar. De hecho, este ha sido el gran sector que ha venido a poner freno a las exigencias que, tanto desde el punto de vista de los servicios como de las ocupaciones requeridas, se han podido desarrollar en él. La contratación formal del trabajo de la mujer -un claro signo de modernidad- se ha debido en gran parte al desarrollo de estas actividades; así mismo, un número importante de la mano de obra más formada ha podido encontrar en estas actividades la oportunidad para su desarrollo.

El segundo de los aspectos que se observa es que está emergiendo una estructura productiva relacionada con el comercio y el sector informal en general. Este sector comercial e informal viene a ocupar una mano de obra que gran parte de las veces no tiene oportunidades de empleo estable. Gentes venidas del campo, población residente en las ciudades que no encuentra una ocupación de forma regular, jóvenes desocupados con un futuro incierto, inmigrantes retornados que empiezan a $\triangleright$ 
observar que el comercio puede ser un medio para vivir y progresar, etcétera, son los que conforman este gran volumen de población. Se estima que la ocupación informal en Argelia se sitúa en torno al 40 por 100 de la ocupación de la mano de obra no agrícola (Lassassi et al, 2015) siendo a su vez el sector que más rápidamente ha crecido en los últimos tiempos. El auge de los pequeños negocios, las pequeñas empresas de servicios, y sobre todo el crecimiento de las empresas relacionadas con el sector de comercio, son las que en este conjunto de actividades están teniendo un gran protagonismo; se afirma que un tercio de los nuevos empresarios argelinos son comerciantes (Hammouda et al, 2008). Sin embargo, ello no quiere decir que ese comercio se derive de una producción interna, no tiene efectos sobre la estructura de producción en tanto que no genera un efecto multiplicador sobre la producción interna porque se está importando aquello que se está comerciando.

$Y$ en tercer lugar, lo que se observa en la estructura productiva es el arrastre de lo que eran las actividades industriales tradicionales, las relacionadas con la extracción de gas y petróleo (Escribano et al, 2007). Ciertamente estas actividades han representado para Argelia los sectores más potentes y seguros; como ya se ha indicado anteriormente, han llegado a significar el 50 por 100 de su PIB y de la ocupación industrial. En la actualidad, y aunque continúan teniendo gran importancia, están perdiendo protagonismo (Martín Muñoz, 1993). Las mejoras en la productividad en cuanto a extracción y tratamiento de aquellos recursos, y la aparición de otras actividades productivas, como se ha apuntado, hacen que la preponderancia que pudieran tener en otros momentos, en la actualidad sea cuestionada.

\section{Las vías y los caminos futuros por hacer}

Entonces, en el marco de alcanzar una diversificación y modernización es donde están puestas las pretensiones del país. En este sentido se han diseñado desde el año 2010 dos planes quinquenales de desarrollo (2010-2014; 2015-2019) que tienen como objetivo el poder cumplir estos propósitos (ver los documentos citados por la Casa Árabe así como por la Embajada de Argelia). Fundamentalmente, ambos planes van en la misma dirección: dotar al país de aquellas infraestructuras sociales y colectivas que permitan mejorar el nivel de vida de la población y en las que paralelamente se pudiese llegar a desarrollar la actividad privada. Básicamente se está hablando de llevar a la práctica un plan de edificación de viviendas (2 millones en el primer plan y 1,6 en el segundo), $1.000 \mathrm{Km}$ de autopistas, $7.000 \mathrm{Km}$ de carreteras, $6.000 \mathrm{Km}$ de líneas ferroviarias, 10 plantas desalinizadoras, 60 centros de tratamiento de aguas, 60 hospitales, 70 maternidades, 100 centros de salud, 3.000 escuelas primarias, 1.000 colegios, etcétera; todo ello unido a la realización de puertos y aeropuertos que permitirán una mayor y mejor comunicación entre las distintas zonas del país, así como con el exterior. Todas estas acciones van encaminadas obviamente a dotar de un equipamiento social que permita trasladar a la población en general los beneficios derivados de las rentas energéticas. Así mismo, las infraestructuras sociales diseñadas van a facilitar el que se inicien, desarrollen o consoliden actividades que vayan a posibilitar la diversificación industrial que está necesitando.

En este marco de cambios, tres serían las actividades que con cierta atención se tratan de desarrollar. En primer lugar las energías alternativas en tanto que existe la voluntad de que, hacia el año 2050, Argelia deje de depender de las energías tradicionales; de ahí la intención de llevar a cabo planes de inversión en energía eólica y solar (por eso el gran interés en las experiencias españolas al respecto). El segundo grupo de interés se centra en el sector turístico, ya que Argelia tiene recursos turísticos de gran atractivo (algunos de ellos Patrimonio de la Humanidad), costas y playas, por lo que, objetivamente, es un país con una capacidad turística semejante a la de sus vecinos (Marruecos y Túnez), pero su nivel de atracción está hoy a un $\triangleright$ 
cuarto respecto de ellos. Y en tercer lugar, existe un interés particular en lo que es el sector primario, la agricultura, ya que es un sector con gran potencial pero muy poco desarrollado en la actualidad.

Sin embargo, a pesar de sus políticas deliberadas y de los recursos que para ello pueda disponer, tres aspectos vienen a limitar el que se estén llevando a cabo estas transformaciones con la celeridad que se requeriría. La primera dificultad se aprecia en cuanto a la inseguridad que se percibe. El número de incidentes terroristas internos que sufre Argelia anualmente es de 27,17 frente a un promedio mundial de 7,5 (Bandyopadhyay et al, 2015); ello hace que tanto la inversión extranjera como la ayuda exterior se aminoren de forma significativa. Así, la inversión extranjera en Argelia es de un 0,76 por 100 del PIB, frente a un 2,90 por 100 en el conjunto mundial. De la misma manera, la ayuda exterior para Argelia es de un 0,6 del PIB, frente al 6,74 que supone para la media a nivel mundial. Esto significa que las inversiones exteriores huyen de una amenaza terrorista que puede estar latente en el ambiente. Junto a ello, el segundo de los problemas con que se encuentra el desarrollo de la economía argelina es que si bien la eficiencia de la inversión pública es baja, el verdadero impedimento es que el crecimiento del sector privado está obstaculizado por un clima complicado de negocios, con un sector financiero poco desarrollado y con una integración internacional limitada. Pese a que se pueden encontrar oportunidades derivadas de tener una mano de obra con salarios que oscilan entre los 180 y 700 euros al mes (frente a los 1.000-1.500 euros pagados en España) (ver información de Planes Quinquenales citados anteriormente), con un nivel de preparación suficiente como para llevar a cabo una actividad, la excesiva burocratización y rigidez en las relaciones con la Administración hacen desistir de llevar a cabo acuerdos con ella. Y, ya por fin, nos encontramos con un tercer problema, y es el intrincado laberinto jurídico que la gobernanza -muchas veces, y con cierta frecuencia, cambiante- exige en este tipo de países. Ello hace que la inseguridad jurídica a la que se está sometido haga renunciar a proceder a llevar a cabo cambios, los cambios que como se han apuntado se necesitan para que se pueda desarrollar el país. Pese a todo, Argelia es un país que más temprano que tarde se verá integrado en el conjunto de la economía mundial con un nivel de desarrollo positivo derivado de los recursos que posee.

\section{Conclusiones: ¿un diseño inacabado?}

Muy posiblemente las oportunidades económicas que Argelia tiene para desarrollarse son muchas. La existencia de recursos estratégicos de primer orden para la economía mundial, y la potencialidad de un mercado interno como el que posee, con expectativas de crecimiento muy resaltables, serían elementos suficientes para pensar de forma favorable. Pero es cierto que existen dificultades para que esto se produzca de manera automática. Algunos de los elementos que muestran los conflictos a los que podemos aludir serían: los desequilibrios poblacionales entre el interior y la costa, la existencia de una cultura económica anclada en los favores personales y políticos de los grupos dominantes, la considerable extensión de una economía informal creciente, la inexistencia de un sector industrial mínimamente estructurado para hacer frente a sus propias necesidades, la desocupación de una mano de obra joven y formada pero sin perspectivas de trabajo, un sector comercial absorbente artificial de las expectativas de expansión de la economía, etcétera.

Por otra parte, se percibe que Argelia está aprovechando las posibilidades económicas de una economía fundamentada en la existencia de recursos energéticos. Es evidente que las piensa explotar con intensidad, de ahí los planes de desarrollo diseñados, que son generosos y ambiciosos. Sin embargo, sin que se llegue a advertir el soporte teórico y práctico en el que pueda estructurarse el cambio previsto, y que vendría inducido en la economía argelina por los planes diseñados, lo que $\triangleright$ 
se aprecia es que no se sabe cuáles van a ser los efectos de los cambios previstos en sus estructuras productivas futuras. El hecho de que se deban importar tanto las tecnologías como parte de los conocimientos para proceder a los cambios previstos, deja en entredicho los efectos internos que se podrían tener para que realmente se hablase de un modelo de desarrollo. De esta forma, los agentes externos podrían ser quienes se beneficiaran de los cambios que se pretenden hacer.

Otra cosa muy distinta será el crecimiento previsto derivado de los planes diseñados. No cabe duda que va a tener una repercusión en cuanto a los estándares de consumo y los productos ofertados. Sin embargo, está por demostrar que vaya a tener una repercusión positiva en lo que corresponde a sus estructuras de producción.

De todo lo anterior, destaca un efecto inductor de cambio, positivo en unos casos, y en otros no tanto, por el hecho de que las propensiones al consumo, a la inversión y, sobre todo, en lo que corresponde a sus capacidades de producción, van a verse modificadas. No obstante, lo verdaderamente importante se centra en lo que pueda derivarse de la mejora en sus modos institucionales de operar. Esto es, el que se alcance una estabilidad y una seguridad en las relaciones con la Administración, con el poder político, con los agentes institucionales -locales y nacionales-, es lo que verdaderamente representaría que los planes de desarrollo van a tener un futuro esperanzador y efectivo.

\section{Bibliografía}

[1] AISSAOUI, A. (2001). Algeria: the political economy of oil and gas, vol. 5. Oxford: Oxford University Press.

[2] BANCO MUNDIAL. Indicadores del desarrollo mundial, Argelia, varios años.

[3] BANDYOPADHYAY, S.; SANDLER, T. y YOUNAS, J. (2015). «El costo del terrorismo«, Finanzas \& Desarrollo, Fondo Monetario Internacional, junio de 2015, vol. 52, n² 2, pp. 26-28.

[4] BARDAK, U. (2013). Youth employment: challenges and policy responses in the Arab
Mediterranean countries. Turin: European Training Foundation.

[5] BARDAK , U. (2015). «Desempleo juvenil y políticas de empleo". AFKAR/IDEAS, n 46, pp. 56-58 (verano 2015).

[6] CASA ARABE (2010). «Argelia: plan quinquenal y nuevas oportunidades para las empresas españolas». Boletín de Economía y Negocios, n 16, p. 3.

[7] ESCRIBANO, G. y LORCA, A. (2007). «Reforma económica en el Magreb: de la estabilización a la modernización». Documento de Trabajo, $\mathrm{n}^{\circ} 3$, Real Instituto Elcano.

[8] HAMMOUDA, N.E. y LASSASSI, M. (2008). «Potentiel entrepreneurial en Algérie: Entre capital humain et informalité, quelle croissance?». Economic Research Forum Working Papers, $n^{\circ} 433$, p. 23.

[9] IMF (2014). «Executive Board Concludes. Article IV Consultation with Algeria». Press Release $\mathrm{n}^{\circ}$. 14/566, december 11, 2014.

[10] LASSASSI, M. y HAMMOUDA, N.E. (2015). «50 ans d'independance: Quelle evolution de la situation du marche du travail en Algerie?». Les cahiers du CREAD, $\mathrm{n}^{\circ} 100$ (enero 2012) pp. 101-136.

[11] MARTíN MUÑOZ, G. (1993). «Argelia, la resistencia al cambio». Anuario Internacional CIDOB 1992, pp. 451-459. Barcelona: Fundación CIDOB.

[12] MOUFFOK, G. (2001). «Argelia: ¿lucha de clanes o lucha de clases?», Le Monde Diplomatique, edición española, julio, pp. 4-5.

[13] UNITED NATIONS DEVELOPMENT PROGRAM (2011). Arab Development Challenges Report. Towards the Developmental State in the Arab Region. Boulac, Cairo, Egypt.

\section{Enlaces de interés:}

[1] Banco de Argelia. Disponible en: http://www.bank-of-algeria.dz/

[2] Cámara de Comercio Hispano-Argelina. Disponible en: http://camarahispanoargelina.com/index. php/es/

[3] Embajada de Argel (Madrid). Disponible en: http://www.emb-argelia.es/economiaargeli nacifras.htm

[4] Ministerio de Finanzas de Argelia. Disponible en: http://www.mf.gov.dz/ 